\title{
ANÁLISE DA ESCALA TEMPORAL DE MONITORAMENTO DAS SECAS AGRÍCOLAS E METEOROLÓGICAS NO ESTADO DE SÃO PAULO
}

\author{
GABRIEL CONSTANTINO BLAIN ${ }^{1}$ E ORIVALDO BRUNINI ${ }^{2}$
}

\author{
1,2 Agência Paulista de Tecnologia dos Agronegócios (APTA), Instituto Agronômico de Campinas (IAC). \\ Av. Barão de Itapura, nº 1487, Botafogo, Campinas, SP, CEP 13001-970, C.P 28 \\ E-mails: ${ }^{1}$ gabriel@iac.sp.gov.br; ${ }^{2}$ brunini@iac.sp.gov.br
}

Recebido Novembro 2005 - Aceito Agosto 2006

\begin{abstract}
RESUMO
$\mathrm{O}$ monitoramento dos diversos tipos de seca vem sendo baseado em índices que os padronizam em escalas temporal e espacial. A confiabilidade dos resultados desses modelos matemáticos, na quantificação das implicações dessas anomalias climáticas, nos diferentes setores da economia, está associada à escolha do correto intervalo de tempo em que cada conceito de seca deve ser avaliado. Por meio das médias mensal e decendial de precipitação pluvial e seus respectivos desvios padrões, do teste Kolmogorov-Smirnov e da comparação entre valores dos índices e parâmetros do balanço hídrico, este estudo demonstrou a importância da adoção da escala adequada de tempo no uso dos índices de seca meteorológica (Índice de Severidade de Seca de Palmer adaptado às condições climáticas do estado de São Paulo, PDSI adap e Índice Padronizado de Precipitação, SPI) e agrícola (Índice Padronizado de Evapotranspiração Real, IPER). Enquanto o estudo das implicações meteorológicas é restrito a escalas temporais maiores ou iguais à mensal, é recomendado que a seca agrícola seja avaliada na escala decendial com base em elementos ligados diretamente à evapotranspiração real das culturas e que, mesmo nesses intervalos temporais, seja estabelecido um valor esperado e válido do parâmetro, para que uma deficiência no suprimento hídrico das culturas seja detectada.
\end{abstract}

Palavras-chave: anomalia climática, índice de seca, deficiência hídrica.

\begin{abstract}
ANALYSIS OF THE TIME PERIOD OF METEOROLOGICAL AND AGRICULTURAL DROUGHT MONITORING IN THE STATE OF SÃO PAULO, BRAZIL.

The monitoring of each type of drought has been based on index that attempt to encapsulate then on regional and temporal basis. The results of those mathematical models on drought implications are associated with choosing the correct time scale that each drought concept should be analyzed. The study was based on monthly and ten day period precipitation average and its standard deviation, and the Kolmogorov-Smirnov test. The paper also compared the index departures with climate water balance parameters. The importance of using the correct time scale, when meteorological drought index (PDSI adap and SPI) or agricultural drought index (IPER) are been used was shown. While the study of the meteorological drought implications has to be done on times scales equal or bigger than the monthly, the agricultural impacts, has to be analyzed based on parameters that are directly associated with actual evapotranspiration of the crops and, even on shorts times scales, the establishment of an expected value of those parameters, that allow the quantification of an crop water surplus deficit, could be detected.
\end{abstract}

Keywords: drought index, water surplus deficit, actual evapotranspiration. 


\section{INTRODUÇÃO}

Um passo importante na análise científica do fenômeno seca foi o reconhecimento das diferentes percepções que diversos setores da sociedade possuem em relação a essa anomalia climática. Essa evolução resultou na definição de tipos de seca, entre os quais pode-se citar: a agrícola, a meteorológica, a hidrológica e a socioeconômica.

Uma conseqüência direta desse fato é a necessidade da adoção de uma escala temporal adequada ao monitoramento de cada tipo de seca. A meteorológica, por exemplo, é definida por Wilhite (2000) como o resultado de um déficit de precipitação, isto é, quando o valor de chuva acumulado em um período e em uma área encontra-se significativamente abaixo do valor climatologicamente esperado.

Com isso, a tentativa de monitoramento da seca meteorológica em intervalos de tempo menores que o mensal (10 dias, por exemplo) poderá resultar em informações inconsistentes, pois devido a sua grande variabilidade temporal, a determinação de um patamar esperado (e consistente) que sirva como base para o cálculo de um possível déficit (anomalia) de precipitação torna-se impraticável.

Em outro aspecto, de acordo com a Organização Meteorológica Mundial (1975), a seca agrícola está relacionada à baixa disponibilidade de umidade no solo, que torna o suprimento de água às culturas insuficientes para repor as perdas por evapotranspiração. Segundo Palmer (1968) o monitoramento desse tipo de fenômeno deve ser baseado no déficit entre o valor da evapotranspiração real (ETR) estimada em um período, e o climatologicamente esperado para o intervalo de tempo e região homogênea em análise. Nesse sentido, o uso da escala mensal para monitorar as implicações da seca agrícola representa uma espera excessivamente longa podendo, com isso, resultar em um elevado déficit de ETR, e, conseqüentemente, em grandes perdas agrícolas. Fica evidente, portanto, que esse tipo de anomalia exige uma escala de tempo detalhada (10 dias, por exemplo) para que seus efeitos sejam corretamente avaliados e para que medidas de controle e mitigação sejam adotadas no momento propício (Palmer, 1968 e Hayes et al., 1999).

Atualmente, o monitoramento dos diversos tipos de seca é feito com base em índices que os padronizam em escala temporal e regional permitindo, com isso, a comparação das condições hídricas de diferentes áreas. Tais quantificadores hídricos vêm sendo usados em políticas estaduais e federais como parâmetros que auxiliam a tomada de decisão quanto à adoção ou suspensão de planos de combate às implicações da seca nos diversos setores da sociedade. O Índice de Severidade de Seca de Palmer (PSDI) e o Índice Padronizado de Precipitação (SPI) são os quantificadores mundialmente mais conhecidos e utilizados. Diversos trabalhos científicos, como por exemplo,
Aley (1984), Karl (1986), Hayes et al. (1999), Gutmam (1998, 1999), Sansigolo (2004) e Blain (2005), discutem suas características e limitações.

A despeito de todas as análises estatísticas realizadas nesses modelos matemáticos, questões conceituais como, por exemplo, o entendimento que índices meteorológicos são restritos à escalas maiores ou iguais à mensal e, portanto, distantes dos interesses agrícolas, permanecem ainda sem um real esclarecimento. Um exemplo desse fato é dado em Aley (1984), quando diz que o PDSI é usualmente calculado em base mensal e semanal.

No cálculo dessa ferramenta, o elemento de maior importância é a cafec precipitation, que representa o valor de chuva esperado ou apropriado para manter uma região sob condições hídricas normais (Palmer, 1965). Tal fator é usualmente calculado com base na média aritmética dos parâmetros do balanço hídrico de Thornthwaite e Mather (1955) como a precipitação, o excedente e a alteração hídrica para o período e região em estudo. Nas escalas semanais ou decendiais, o elevado valor do desvio padrão de tais médias, especialmente no caso da chuva, evidencia que a estimativa da cafec precipitation carece de consistência e, portanto, não deve ser usada como patamar esperado de precipitação. Nesse sentido, o uso do PDSI ou do SPI para a avaliação da seca agrícola ou meteorológica nessas escalas resultará em informações com baixa confiabilidade.

Com isso, este estudo tem como objetivo demonstrar a importância da adoção da escala de tempo adequada para a avaliação e monitoramento tanto da seca meteorológica quanto da agrícola por meio do correto uso dos índices meteorológicos (Índice Padronizado de Precipitação e Índice de Severidade de Seca de Palmer adaptado ao estado de São Paulo, PDSI adap) e agrícola (IPER) no estado de São Paulo.

\section{MATERIAL E MÉTODOS}

A eficiência dos índices nas diferentes escalas temporais será ilustrada por meio de dados de temperatura do ar e precipitação pluviométrica dos postos meteorológicos de Campinas, SP (22 $54^{\prime}$ 'S; $47^{\circ} 05^{\prime} \mathrm{W}$; 669m) e Pindorama ( $22^{\circ} 13^{\prime}$ 'S; $48^{\circ} 56^{\prime} \mathrm{W}$; $562 \mathrm{~m})$ pertencentes ao Instituto Agronômico da Secretaria de Agricultura e Abastecimento entre os anos de 1971 a 2004. De acordo com a classificação de Köppen, esses municípios apresentam, respectivamente, os climas tropical de altitude (Cwa) e de savanas (Aw) sendo, esses dois tipos climáticos, observados em grande parte dos municípios do estado. Com isso se uma determinada escala temporal monstrar-se inadequada para monitorar um tipo de seca nesses postos, pode-se assumir que tal intervalo de tempo não é recomendado para avaliação padronizada desse fenômeno climático no estado de São Paulo. Os balanços hídricos foram calculados de acordo com o método 
de Thornthwaite e Mather (1955), por meio de software desenvolvido por Brunini e Caputti (2002).

O SPI e o PDSI adap foram calculados como descritos nos trabalhos de McKee et al. (1993) e Blain (2005) nas escalas mensais e decendiais. O IPER, desenvolvido por Blain e Brunini (2005), é calculado da seguinte forma: a primeira etapa para a estimativa desse quantificador é a determinação da função densidade de probabilidade beta, que descreve uma longa série de valores estimados da ETR decendial. Na seqüência, a probabilidade cumulativa de um dado patamar de ETR é calculada. A função normal inversa (gaussiana), que possui média zero e variância unitária, é aplicada à probabilidade acumulada. O resultado é o valor do Índice Padronizado de Evapotranspiração Real (IPER). Valores do IPER próximos ou superiores a 0 indicam que a ETR acumulada em um dado decêndio está próxima ou superior ao valor climatologicamente esperado desse parâmetro no período; valores negativos do índice evidenciam que a evapotranspiração real de um dado decêndio está abaixo do patamar esperado e, portanto, a umidade do solo encontra-se insuficiente para repor as perdas por evapotanspiração das culturas.

A distribuição beta é definida no intervalo $(0,1)$ com a seguinte função densidade de probabilidade (PDF) da variável x:

$f(X)=\frac{X^{(\alpha-1)-(1-X)^{\beta-1}}}{\beta(\alpha, \beta)}$ para $0<x>1$

Os parâmetros $\alpha$ e $\beta$ determinam a forma da distribuição e são calculados da seguinte forma:

$\beta=\left[\frac{\overline{\mathrm{x}}(1-\overline{\mathrm{x}})}{\mathrm{S}^{2}}-1\right] *(1-\overline{\mathrm{x}})$

$\alpha=\frac{\bar{x}^{2}(1-\bar{x})}{S^{2}}$

em que:

$\overline{\mathrm{x}}$ a média aritmética dos valores de $\mathrm{x}$ na série considerada,

$\mathrm{S}^{2}$ a variância da série de valores de $\mathrm{x}$ considerada.

Considerando que as temperaturas médias diárias no estado de São Paulo impossibilitam valores de ETR decendiais acima de $100 \mathrm{~mm}$, optou-se pela seguinte transformação de variável para que a PDF beta possa ser aplicada às séries de ETR decendiais, conforme segue:

$\mathrm{ETR} "=\mathrm{ETR} / 100$

G(ETR") é então transformada em uma variável normal (valor final do IPER) por meio das equações desenvolvidas por Abramowitz e Stegun, (1965).

IPER $=+\left(\mathrm{t}-\frac{\mathrm{co}+\mathrm{c}_{1} \mathrm{t}+\mathrm{c}_{2} \mathrm{t}^{2}}{1+\mathrm{d}_{1} \mathrm{t}+\mathrm{d}_{2} \mathrm{t}^{2}+\mathrm{d}_{3} \mathrm{t}^{3}}\right)$ para $0<\mathrm{G}(\mathrm{x}) \leq 0,5$

IPER $=+\left(\mathrm{t}-\frac{\mathrm{co}+\mathrm{c}_{1} \mathrm{t}+\mathrm{c}_{2} \mathrm{t}^{2}}{1+\mathrm{d}_{1} \mathrm{t}+\mathrm{d}_{2} \mathrm{t}^{2}+\mathrm{d}_{3} \mathrm{t}^{3}}\right)$ para $0<\mathrm{G}(\mathrm{x}) \leq 0,5$ em que:

$$
\begin{aligned}
& \mathrm{t}=\sqrt{\left(\ln \left(\frac{1}{(\mathrm{G}(\text { ETR }))^{2}}\right)\right.} \quad \text { para } 0<\mathrm{G}(\text { ETR }) \leq 0,5 \\
& \mathrm{t}=\sqrt{\left(\ln \left(\frac{1}{(1-\mathrm{G}(\text { ETR }))^{2}}\right)\right.} \text { para } 0,5<\mathrm{G}(\text { ETR })<1
\end{aligned}
$$

em que:

$\mathrm{c}_{\mathrm{o}}=2,515517$;

$\mathrm{c}_{1}=0,802853$

$\mathrm{c}_{2}=0,010328$

$\mathrm{d}_{1}=1,432788$

$\mathrm{d}_{2}=0,189269$;

$\mathrm{d}_{3}=0,001308$.

A subtração entre o valor da ETR estimada em um dado período e sua média decendial deu origem ao parâmetro AH_ETR. Esse último elemento foi dividido pelo desvio padrão decendial dos valores da ETR resultando no AH_ETR/DP, que representa um possível desvio (déficit) que um dado valor estimado de ETR apresenta em relação a seu valor esperado, considerando-se as características climáticas do período e da região.

No cálculo do Índice de Severidade de Seca de Palmer adaptado às condições do estado de São Paulo, o elemento que quantifica as condições de umidade em um determinado período é o fator $\mathrm{Z}$ de anomalia hídrica. Assim, para verificar a consistência dos índices apresentados nesse estudo no monitoramento da seca agrícola, o SPI, Z e o IPER, todos na escala decendial, foram comparados à AH_ETR/DP por meio de regressões lineares simples.

A adequação do SPI e do PDSI adap para o monitoramento da seca meteorológica, na escala mensal, foi comprovada em Blain (2005). A fim de avaliar a consistência dessas duas últimas ferramentas no monitoramento de um possível déficit decendial de precipitação, a média (usada no cálculo da cafec precipitation) e o desvio padrão desse elemento meteorológico foram estimados.

O teste Komolgorov-Smirnov (KS) foi aplicado às séries de dez dias de chuva a fim de verificar seu grau de aderência à distribuição gama incompleta (base do modelo do SPI). Segundo Assis et al. (1996), o KS pode ser aplicado para avaliar se os valores de uma série temporal podem ser considerados como provenientes de uma população com distribuição teórica preestabelecida, sob a hipótese da nulidade Ho. O teste é definido por:

$\operatorname{Dmax}=\operatorname{Max}\left|F^{\prime}(\mathrm{x})-\mathrm{F}(\mathrm{x})\right|$

$F(x)=f a /(n+1)$

em que:

Max, Maior valor absoluto entre as classes de freqüência estabelecidas no teste; 
Dmax, Valor calculado do teste do Kolmogorov-Smirnov; $\mathrm{F}^{\prime}(\mathrm{x})$, função distribuição de probabilidade teórica;

$\mathrm{F}(\mathrm{x})$, função distribuição de probabilidade empírica;

$\mathrm{Fa}$, freqüência acumulada de cada classe;

$\mathrm{n}$, número de classes de valores pre-estabelecidos.

Quando o teste do KS for estatisticamente significante (valor de Dmax maior do que o patamar crítico relativo ao nível de significância adotado), a hipótese de que os dados amostrais podem ser ajustados à distribuição teórica pré-estabelecida é rejeitada (nesse estudo foram adotados os patamares de $5 \%$ e 10\% de significância).

\section{RESULTADOS E DISCUSSÃO}

A subtração entre os valores das ETR estimadas em um balanço hídrico seqüencial e as respectivas médias de cada decêndio deu origem ao parâmetro AH_ETR. Esse último elemento foi dividido pelo desvio padrão decendial dos valores da ETR resultando no AH_ETR/DP, que representa um possível desvio (déficit) que um dado valor estimado de ETR apresenta em relação a seu valor esperado, em cada período e região, assim, nota-se que o parâmetro AH_ETR/DP é relacionado à perda por evapotranspiração das culturas (seca agrícola). A alta correlação entre o Índice Padronizado de Evapotranspiração Real (IPER) e esse elemento, demonstrada na Figura 1 na escala decendial, indica que a variação desse índice está diretamente relacionada ao número de desvios padrões que um dado valor de ETR encontra-se abaixo do valor climatologicamente esperado em um período e localidade. Nos casos em que a ETR estimada encontra-se relativamente próxima ao patamar esperado (AH_ETR/DP > -2) a variação do IPER em relação a

Tabela 1 - Variação do Índice Padronizado de Evapotranspiração Real (IPER) em relação ao parâmetro AH_ETR/DP (déficit que um dado valor de ETR estimado em um balanço hídrico seqüencial apresenta em relação a seu valor esperado, em cada período e região) na localidade de Pindorama em casos de seca moderadas e severas.

\begin{tabular}{ccc}
\hline Data & IPER & AH_ETR/DP \\
\hline $11 / 03 / 73$ & $-5,24$ & $-3,92$ \\
$11 / 12 / 88$ & $-5,08$ & $-4,15$ \\
$21 / 02 / 73$ & $-4,51$ & $-3,75$ \\
$21 / 11 / 91$ & $-3,71$ & $-2,78$ \\
$21 / 06 / 03$ & $-1,57$ & $-1,38$ \\
$21 / 04 / 00$ & $-1,51$ & $-1,51$ \\
$21 / 12 / 89$ & $-0,36$ & $-0,38$ \\
$21 / 06 / 91$ & $-0,36$ & $-0,40$ \\
$11 / 07 / 91$ & $-0,36$ & $-0,36$ \\
\hline
\end{tabular}

este último parâmetro permanece junto à reta 1:1. Entretanto, para casos mais extremos de seca, essa variação linear não é observada, como pode ser visualizada com dados da localidade de Pindorama apresentados na Tabela 1.

Ao estimarem o regime do armazenamento hídrico de maneira exponencial, Thornthwaite e Mather (1955) reconhecem que conforme o solo perde sua umidade, a água restante é submetida a uma tensão crescente de retenção, que por sua vez é inversamente proporcional à disponibilidade hídrica às culturas. Com isso, é desejado que um índice de seca agrícola reconheça que uma mesma variação em AH_ETR/DP (parâmetro relacionado a demanda hídrica das culturas) apresenta condições cada vez mais severas de seca conforme a reserva hídrica do solo distancia-se de sua máxima capacidade. Assim, conclui-se que o Índice Padronizado de Evapotranspiração Real pode ser usado para monitoramento e padronização da seca agrícola no estado de São Paulo, permitindo a comparação das condições desse tipo de fenômeno em diferentes áreas e épocas. A correlação entre o SPI, o fator Z e AH_ETR/DP na escala decendial é apresentada na Figura 2.

A baixa correlação evidenciada na Figura 2 entre os índices meteorológicas e AH_ETR/DP demonstra que o SPI que utiliza apenas dados de precipitação - e o Índice de Palmer - que, de acordo com Blain (2005), considera a chuva o elemento
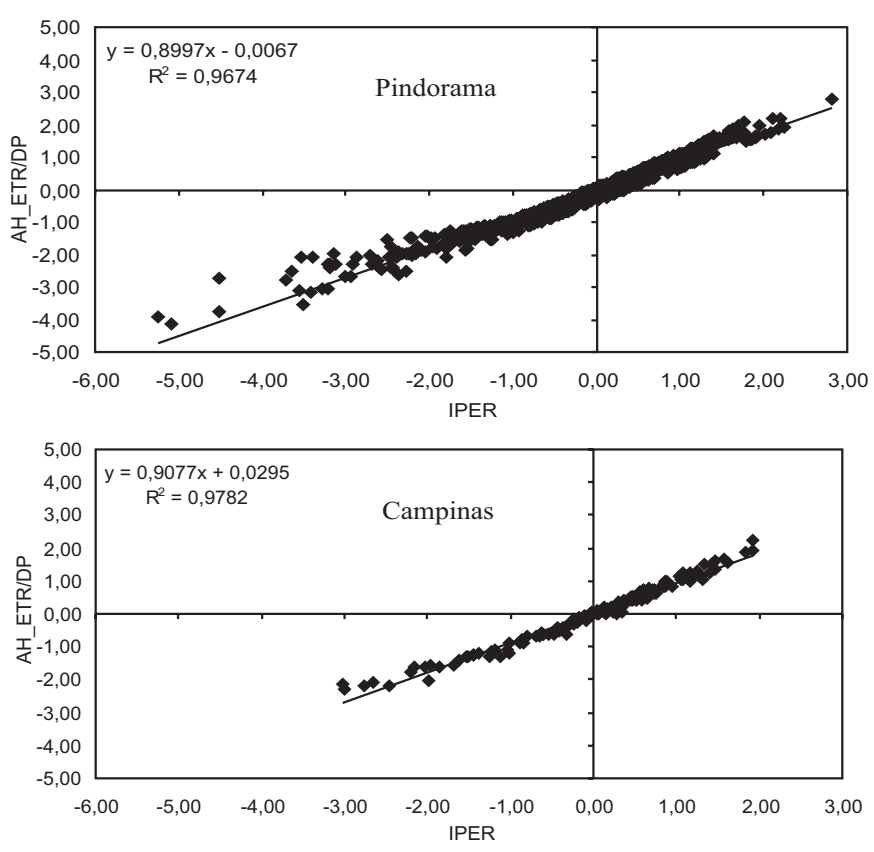

Figura 1 - Regressão linear simples entre AH_ETR/DP (déficit que um dado valor de ETR estimado em um balanço hídrico seqüencial apresenta em relação a seu valor esperado, em cada período e região) e o Índice Padronizado de Evapotranspiração Real (IPER), na escala decendial, entre os anos de 1971 a 2004 nas localidades de PindoramaSP e Campinas-SP 

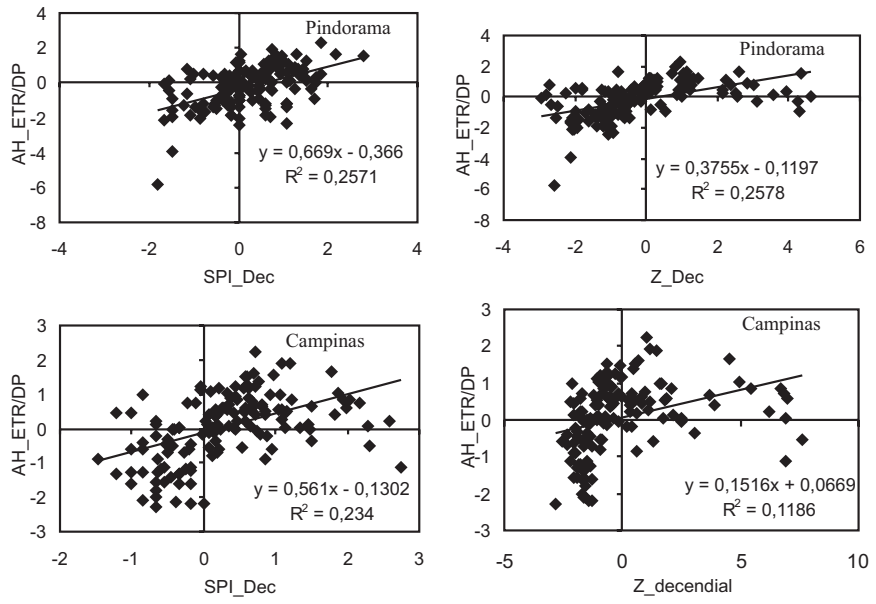

Figura 2 - Regressão linear simples entre AH_ETR/DP (déficit que um dado valor de ETR estimado em um balanço hídrico seqüencial apresenta em relação a seu valor esperado, em cada período e região) e o fator de anomalia hídrica Z e entre AH_ETR/DP e o Índice Padronizado de Precipitação (SPI), na escala decendial, entre os anos de 1971 a 2004 nas localidades de Pindorama-SP e Campinas-SP.

de maior importância em seu cálculo - não são adequados para monitorar as perdas por evapotranspiração de uma cultura, mesmo que calculados em base de 10 dias. Torna-se evidente, portanto, que o início de uma seca agrícola pode distanciar-se do de uma meteorológica, pois depende quase que exclusivamente das fases de grande demanda hídrica das culturas e da água disponível na zona radicular do solo.

Na Figura 3 são comparados os valores decendiais médios de precipitação com seus respectivos desvios padrões nas localidades de Campinas e Pindorama:
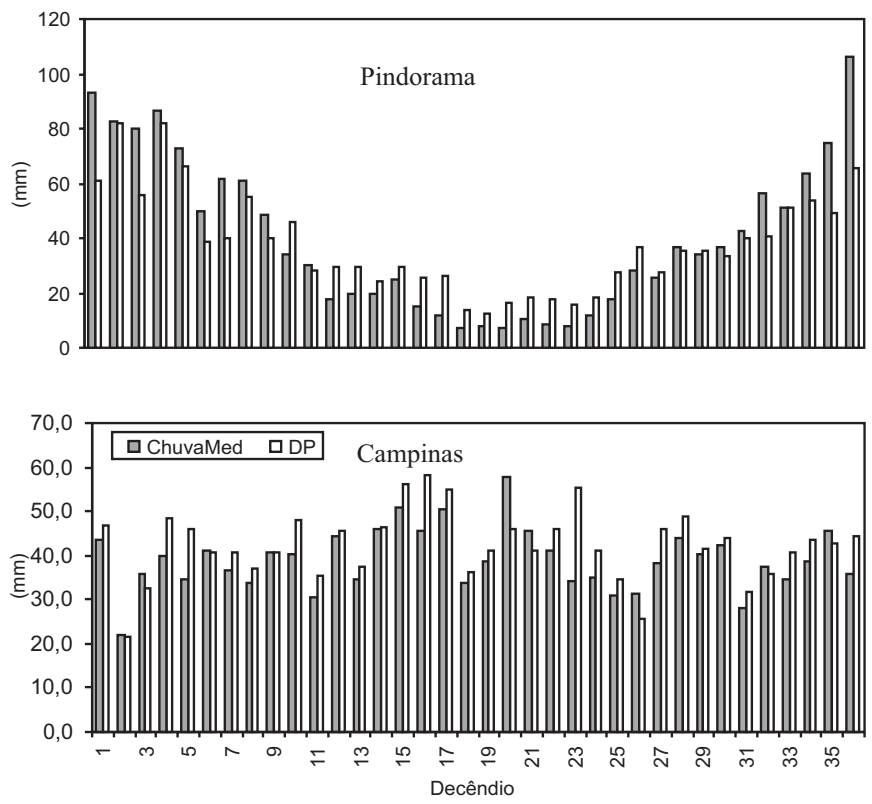

Figura 3 - Médias decendiais de precipitação (1971 a 2004) e seus respectivos desvios padrões em Pindorama-SP e Campinas-SP
Os altos valores dos desvios padrões apresentados na Figura 3 demonstram que o uso da média aritmética de precipitação decendial carece de consistência não podendo, portanto, ser utilizada para o cálculo de um possível déficit de precipitação e, conseqüentemente, para o estudo da seca meteorológica. Tal fato, também inviabiliza a estimativa da cafec precipitation.

Uma das principais etapas do modelo do SPI é o ajuste das séries de precipitação à distribuição gama incompleta, que é indefinida para o valor zero. Em alguns decêndios de junho, julho e agosto, o número de ocorrência de períodos com valores nulos de precipitação pode superar o de observações com registro de chuva, inviabilizando dessa forma, o cálculo da PDF gama (parâmetros de forma e escala apresentariam valores negativos, de acordo com o método da máxima verossimilhança) e conseqüentemente do Índice Padronizado de Precipitação. Ainda nesse aspecto, o teste KS, demonstrado na Figura 4, indica que mesmo em épocas com baixa ocorrência de valores nulos de precipitação (terceiro decêndio de setembro) a distribuição gama incompleta não pode ser aplicada.

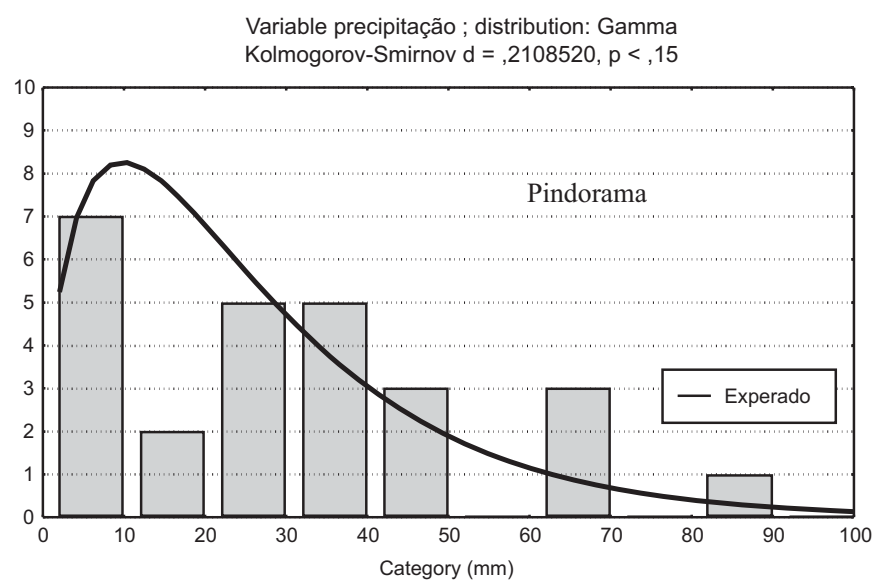

Variable precipitação ; distribution: Gamma Kolmogorov-Smirnov d = ,2354543, $p<, 10$



Figura 4 - Teste Kolmogorov-Smirnov para séries decendiais de precipitação ajustadas por meio da função densidade de probabilidade gama no terceiro decêndio de setembro nas localidades de Pindorama e Campinas. Valores esperados representados em linha e observados em coluna. 
Para Campinas, o valor de KS 0,235 indica diferença significativa, a $5 \%$ de probabilidade, entre a freqüência de precipitação observada e a esperada. Em outras palavras, há apenas $5 \%$ de possibilidade dos dados decendiais de chuva ajustarem-se à distribuição gama (erro tipo 1 , rejeição de Ho verdadeiro). Analogamente, em Pindorama, o valor de 0,2108 indica diferença significativa, a $10 \%$ de probabilidade com isso, há apenas $10 \%$ de possibilidade dos dados ajustarem-se a distribuição gama. Assim, torna-se inviável o uso da PDF gama e conseqüentemente do SPI no monitoramento de um possível déficit decendial de precipitação.

\section{CONCLUSÃO}

Índices que quantificam a seca sob o ponto de vista meteorológico (SPI e PDSI adap) são restritos a escalas temporais maiores ou iguais à mensal. Em pequenos intervalos de tempo (10 dias, por exemplo), a grande variabilidade temporal da precipitação dificulta o estabelecimento de um patamar esperado e consistente, que possa ser usado no cálculo de uma possível anomalia ou déficit. A tentativa de estimativa decendial dessas ferramentas resultará em valores numéricos que não apresentam correlação com parâmetros da seca agrícola ou meteorológica.

A seca, sob o enfoque agronômico, deve ser avaliada em escala decendial com base em elementos ligados diretamente à evapotranspiração real das culturas e que, mesmo nesses intervalos temporais, seja possível estabelecer um valor esperado e válido do parâmetro, para que uma deficiência no suprimento hídrico das culturas seja monitorada e detectada.

\section{AGRADECIMENTOS}

Os autores agradecem a colega Lucí Hidalgo Nunes do Instituto de Geociências da UNICAMP pelas sugestões e leitura do presente trabalho.

\section{REFERÊNCIAS BIBLIOGRÁFICAS}

ABRAMOWITZ, M.; STEGUN., I.A. Handbook of mathematical function, Dover, 1965.

ALLEY, W.M. The Palmer drought severity index: limitations and assumptions. Journal of Climate and Applied Meteorology, v.23, p. 1100-1366, 1984,

ASSIS, F. N.; ARRUDA, H.V.; PEREIRA, A.R. Aplicações de estatística `a climatologia. Pelotas, Universitária, 1996.
BLAIN, G. C. Avaliação e adaptação do Índice de Severidade de Seca de Palmer (PDSI) e do Índice Padronizado de Precipitação (SPI) às condições climáticas do Estado de São Paulo. 2005. 120f. Dissertação (Mestrado em Agricultura Tropical e Sub-tropical) - Instituto Agronômico, Campinas $-\mathrm{SP}$.

BLAIN, G. C., BRUNINI, O. Quantificação da seca agrícola pelo Índice Padronizado de evapotranspiração Real (IPER) no estado de São Paulo. Bragantia, v.65, n.3, p. 519-527, 2005 .

BRUNINI, O., CAPUTTI, E. Balanço hídrico seqüencial para o Estado de São Paulo-Software. 2002.

GUTTMAN, G.B. Comparing the Palmer Drought Index and the Standardized Precipitation Index. Journal of the American Water Resources, v.16, n.2, p. 113-121, 1998.

GUTTMAN, G.B. Accepting the Standardized Precipitation Index: A calculation algorithm. Index. Journal of the American Water Resources, v.35, n.2, p. 311-322, 1999.

HAYES, M. J., SVOBODA, M.D., WILHITE, D.A., VANYARKHO, O.V. Monitoring the 1996 drought using the Standardized Precipitation Index. Bulettin of the American Meteorological Socirty,Nebraska, v.80, n.3, p.429-438, 1999.

KARL, T. The sensitivity of the Palmer Drought Severity Index and Palmer's Z-index to their calibration coefficients including potencial evapotranspiration. Journal of Climate and Applied Meteorology, v.25, p. 77-86, 1986.

MCKEE,T.B., DOESKEN, N.J., KLEIST,J. The relationship of drought frequency and duration to times scale. In: CONFERENCE ON APPIED CLIMATOLOGY, 8.,1993, Boston. Anais..., Boston: PREPRINTS, 1993. p.179-184.

ORGANIZAÇÃO METEOROLÓGICA MUNDIAL: Drought and agriculture. WMO Tech. Note 138, Publ. WMO-392, Geneva, Switzerland, 127p. 1975.

PALMER, W.C. Keeping track of crop moisture conditions, nationwide: the new Crop Moisture Index, Weatherwise, v.21, p.156-161, 1968.

PALMER, W.C. Meteorological drought. US Weather Bureau Res. $\mathbf{N}^{\mathbf{0}} \mathbf{4 5}$. Washington, 58p, 1965. 
SANSIGOLO, C.A. Análise comparativa do desempenho dos Índices de Seca de Palmer (PDSI) e da Precipitação Normalizada (SPI) em Piracicaba, SP (1917-2001). Revista Brasileira de Meteorologia, v.19, n.1, p. 51-56, 2004.

THORNTHWAITE, C.W., MATHER, J.R. The water balance. Climatology, v.8, n.1, p.1 - 40, 1955.
WILHITE, D. A., Drought as a natural hazard: Conceptions and definitions. In: WILHITE, D. A. Drought : A global assessment. Nebraska: Routledge, 2000. p. $111-120$. 